\title{
Conhecimento da pessoa com transtornos mentais sobre o tratamento medicamentoso
}

Knowledge related to drug therapy among people with mental disorders

Conocimiento de la persona con trastornos mentales sobre el tratamiento medicamentoso

\section{Camila Bonfim de Alcantara ${ }^{\mathrm{I}}$, Aline Cristina Zerwes Ferreira ${ }^{\mathrm{II}}$, Fernanda Carolina Capistrano $^{\mathrm{III}}$, Manuela Kaled ${ }^{\mathrm{IV}}$, Caio Cézar da Fonseca Vale ${ }^{\mathrm{V}}$, Mariluci Alves Maftum ${ }^{\mathrm{VI}}$}

Resumo: Objetivo: identificar o conhecimento da pessoa com transtorno mental sobre o tratamento medicamentoso. Método: pesquisa observacional transversal desenvolvida com 300 pessoas em tratamento em dois Centros de Atenção Psicossocial de Curitiba, Paraná. Os dados foram coletados por entrevista estruturada e consulta à prescrição de medicamentos em prontuário. Foi realizada análise descritiva e inferencial. Resultados: a maioria dos participantes apresentou conhecimento insuficiente quanto ao nome dos medicamentos (64\%), dose (91,9\%) e frequência de administração (82,6\%). Houve associação entre conhecimento insuficiente sobre a terapêutica medicamentosa e uso de medicamentos para comorbidades, desconhecimento do diagnóstico, depressão, idade avançada, autoadministração dos medicamentos, consumo de álcool e informações fornecidas pela enfermagem ou psicólogos. Conclusões: destaca-se que, principalmente, a autoadministração de medicamentos, transtornos depressivos e uso de medicamentos para outras condições de saúde podem estar relacionadas ao nível de conhecimento insuficiente da terapêutica medicamentosa, representando possíveis obstáculos a adesão e uso seguro dos medicamentos.

Descritores: Transtornos mentais; Prescrições de medicamentos; Conhecimento do paciente sobre a medicação; Serviços de saúde mental

\footnotetext{
${ }^{\text {I }}$ Acadêmica de enfermagem e membro do grupo de pesquisa Núcleo de Estudo, Pesquisa e Extensão em Cuidado Humano em Enfermagem (NEPECHE). Universidade Federal do Paraná (UFPR). Curitiba, Paraná, Brasil. E-mail: cbalcantara47@gmail.com. Orcid: https://orcid.org/0000-0001-8175-9569

II Enfermeira. Doutoranda do Programa de Pós-graduação em Enfermagem da UFPR (PPGENF-UFPR) e, membro do grupo de pesquisa NEPECHE. UFPR. Curitiba, Paraná, Brasil. E-mail: alinezerwes@gmail.com. Orcid: https://orcid.org/0000-0002-0038-1021

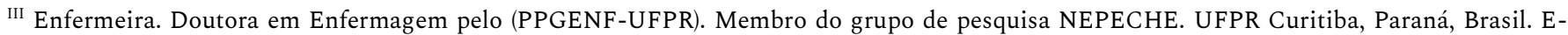
mail: Fernanda_capistrano@yahoo.com.br Orcid: https://orcid.org/0000-0002-2078-5007

IV Enfermeira. Mestranda do PPGENF-UFPR e, membro do grupo de pesquisa NEPECHE. UFPR. Curitiba, Paraná, Brasil. E-mail: manuelakaled@gmail.com. Orcid: https://orcid.org/0000-0001-9760-1029

V Enfermeiro. Mestrando do PPGENF-UFPR e, membro do grupo de pesquisa NEPECHE. UFPR. Curitiba, Paraná, Brasil. E-mail: enf.caiocezar@hotmail.com. Orcid: https://orcid.org/0000-0002-5694-0988

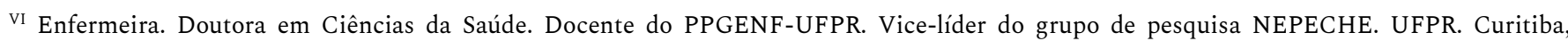
Paraná, Brasil. E-mail: Maftum@ufpr.br. Orcid: https://orcid.org/0000-0001-8706-7299
} 


\begin{abstract}
Objective: to identify knowledge about drug therapy among those people with mental illness. Method: cross-sectional observational research carried out with 300 people undergoing treatment at Psychosocial Care Centers in Curitiba, Paraná. Data were collected through structured interviews and by consulting prescriptions for medications in medical records. A descriptive and inferential analysis was performed. Results: the majority of participants had insufficient knowledge regarding the name of the drugs (64\%), dose (91.9\%) and frequency of administration (82.6\%). There was an association between insufficient knowledge regarding drug therapy and the use of drugs for comorbidities, ignorance about the diagnosis, depression, advanced age, self-administration of drugs, alcohol consumption and misunderstanding information provided by nurses or psychologists. Conclusions: it is underscored that self-administration of medications, depressive disorders and use of medications for other health conditions may be related to the level of insufficient knowledge regarding their drug therapy; thus representing obstacles to adherence of medications.
\end{abstract}

Descriptors: Mental disorders; Prescriptions for medications; Patient's knowledge about medication; Mental health services

Resumen: Objetivo: identificar el conocimiento de la persona con trastorno mental sobre el tratamiento medicamentoso. Método: investigación observacional transversal desarrollada con 300 personas en tratamiento en Centros de Atención Psicosocial de Curitiba, Paraná. Los datos han sido recolectados por entrevista estructurada y consulta a la prescripción de medicamentos. Ha sido realizado análisis descriptivo e inferencia. Resultados: La mayoría de los participantes ha presentado conocimiento insuficiente cuanto al nombre de los medicamentos (64\%), dosis $(91,9 \%)$ y frecuencia de administración $(82,6 \%)$. Ha habido asociación entre conocimiento insuficiente sobre la terapéutica y uso de medicamentos para comorbilidad, desconocimiento del diagnóstico, depresión, edad avanzada, auto-administración de medicamentos, consumo de alcohol e información provista por la enfermería o psicólogos. Conclusiones: Se destaca que la auto-administración de medicamentos, trastornos depresivos y uso de medicamentos para otras condiciones de salud pueden estar relacionadas al nivel de conocimiento insuficiente de la terapéutica medicamentosa, representando obstáculos de adhesión.

Descriptores: Trastornos mentales; Prescripción de medicamentos; Conocimiento del paciente sobre la medicación; Servicios de salud mental

\title{
Introdução
}

Os transtornos mentais são classificados como condição crônica e de alta prevalência, que acometem pessoas de qualquer classe social e fase da vida. A Organização Mundial da Saúde estima que 650 milhões de pessoas no mundo apresentam algum transtorno mental. ${ }^{1}$ Sendo que para o Brasil esses dados equivalem a aproximadamente 3\% da população acometida por transtornos mentais severos e persistentes, necessitando de atenção contínua em saúde mental. Além disto, cerca de $12 \%$ da população, embora não apresente transtornos mentais severos, necessita de algum atendimento contínuo ou eventual em saúde mental. ${ }^{2}$ 
Os transtornos mentais se caracterizam pela manifestação de importantes alterações na cognição, na regulação emocional e/ou no comportamento da pessoa. ${ }^{1,3}$ A pessoa com transtorno mental pode apresentar significativo comprometimento da percepção da realidade, além de dificuldades no relacionamento interpessoal e familiar, comprometimento do desempenho em atividades cotidianas e perda de envolvimento social. ${ }^{4-6}$ Deste modo, estes transtornos frequentemente estão associados a intenso sofrimento e incapacidade psicossocial. ${ }^{1,3}$

Para tanto, o tratamento em saúde mental requer um processo contínuo a partir de múltiplas intervenções e cuidado multiprofissional. ${ }^{6,7}$ Entre as diversas modalidades terapêuticas, destacase a medicamentosa, pois o uso racional e regular de psicofármacos possibilita o controle das graves alterações decorrentes do transtorno mental, contribuindo para o bem-estar físico e mental, melhora nas relações interpessoais e maior sensação de segurança e autocontrole. ${ }^{4,7}$ Além disso, o controle da sintomatologia por meio do uso de psicofármacos, favorece a participação efetiva da pessoa no tratamento psicossocial, auxiliando na reinserção social e em sua autonomia como cidadão. ${ }^{6,7}$

Entretanto, para alcançar seu potencial terapêutico é fundamental que a pessoa utilize o medicamento de forma regular. ${ }^{8-9}$ Entre os diversos fatores que contribuem para o uso irregular do psicofármaco destaca-se o nível de conhecimento que a pessoa apresenta sobre o medicamento prescrito, haja vista que o desconhecimento a respeito da dose, horários e frequência da administração podem resultar no uso irregular e inadequado do medicamento. ${ }^{7,9}$

Além disto, a falta de conhecimento sobre a indicação terapêutica do psicofármaco, efeitos colaterais e adversos e o tempo de uso do medicamento pode levar a pessoa a questionar a real eficácia e importância do tratamento, de modo a se sentir desmotivado e optar por alterar o esquema terapêutico segundo critérios próprios. ${ }^{7,9-10}$ Deste modo, tornam-se mais susceptíveis a interações medicamentosas e a ter dificuldade em identificar os possíveis efeitos colaterais e 
adversos do medicamento entre outros comportamentos que podem colocar sua segurança em risco..$^{911-13}$

Assim, compreende-se que cumprir a prescrição medicamentosa geralmente está relacionado ao nível de conhecimento da pessoa em relação à sua prescrição. ${ }^{4}$ Considerando isto, a Organização Pan-Americana de Saúde (2016) recomenda que ações de educação sobre fármacos sejam empregadas pelos profissionais da saúde para auxiliar a pessoa a conhecer seu tratamento e compreender intervenções necessárias que são fundamentais para promover o uso racional e seguro de medicamentos. ${ }^{14}$

A construção do conhecimento compreende um processo complexo e subjetivo, no qual a pessoa deve buscar correlacionar as informações recebidas aos aspectos simbólicos e pessoais, relacionados com o meio social e cultural ao qual está inserido. Nesse sentido, o conhecimento não deve se limitar a simples aceitação de informações, pois trata-se de um processo ativo e interpessoal no qual a pessoa transforma a informação na mesma medida em que é transformado por esta. ${ }^{15}$

No entanto, a construção deste conhecimento pela pessoa com transtornos mentais representa um desafio, haja vista que a gravidade dos transtornos e a agudização dos sintomas podem dificultar tanto o processo de compreender informações relacionadas a prescrição de medicamentos, como a capacidade da pessoa ao seguir de forma adequada e autônoma o esquema terapêutico. Ainda, o pouco ou nenhum conhecimento pode estar relacionado à falta de insight sobre o transtorno e a necessidade de tratamento, bem como a percepção de cura frente a remissão da sintomatologia. ${ }^{9,11-12}$

Neste contexto, a equipe de enfermagem possui papel fundamental no tratamento de pessoas com transtornos mentais, podendo atuar minimizando esta lacuna de conhecimento, contemplando em seu processo de cuidado a educação em saúde do usuário e seus familiares de modo 
individualizado ou grupal, atentando para que a construção do conhecimento seja efetiva, respeitando o protagonismo do usuário assim como sua corresponsabilização pelo tratamento. ${ }^{7,9,15}$ Salienta-se que, informações claras e suficientes acerca dos medicamentos prescritos podem ajudar a pessoa a tomar uma posição de apropriação e protagonismo em relação ao seu tratamento. ${ }^{16-17}$ Assim, conhecer a indicação terapêutica, os efeitos benéficos da medicação, bem como os possíveis efeitos colaterais auxilia no processo de tomada de decisão ao optar por dar seguimento a terapêutica ou abandoná-la. ${ }^{7,9,16}$

Diante do exposto, evidencia-se a importância de trabalhos científicos que abordem o conhecimento da pessoa com transtornos mentais acerca do tratamento medicamentoso e os fatores associados a este fenômeno, pois auxilia profissionais de saúde a direcionarem a assistência prestada, objetivando a promoção do conhecimento da pessoa sobre esta temática, aspecto que contribui para uma maior eficácia do tratamento, além de favorecer a adesão ao tratamento psicossocial. Nesta perspectiva a presente pesquisa teve como questão: Qual o conhecimento da pessoa com transtorno mental sobre o tratamento medicamentoso? E, apresenta como objetivo: identificar o conhecimento da pessoa com transtorno mental sobre o tratamento medicamentoso.

\section{Método}

Pesquisa observacional transversal desenvolvida em dois Centros de Atenção Psicossocial (CAPS), que ofertam cuidado a pessoas com intenso sofrimento psíquico decorrente de transtornos mentais, da cidade de Curitiba, Paraná (PR), Brasil. Foram convidadas a participar da pesquisa todas as pessoas com diagnóstico de transtorno mental em tratamento com prescrição medicamentosa e com idade igual ou superior a 18 anos.

Dos 372 usuários que compareceram ao CAPS no período da coleta de dados, foram excluídos 13 que estavam com exacerbação dos sintomas do transtorno mental e 43 que não apresentavam 
condições cognitivas para participar da pesquisa, sustentada pela avaliação da equipe de saúde do serviço; e 14 usuários se recusaram a participar da pesquisa após três tentativas de convite. Por fim, a amostra final contemplou 300 usuários.

A coleta de dados ocorreu de abril a junho de 2014, por entrevista estruturada a partir da aplicação de um instrumento, elaborado pelos pesquisadores, para a caracterização sociodemográfica (sexo, idade, estado civil, escolaridade e situação empregatícia), clínica (diagnóstico de transtorno mental, apresentar outras comorbidades e o uso de medicamentos para tal) e farmacoterapêutica (nome, dose e frequência de uso dos psicofármacos).

Além da entrevista, os dados referentes ao diagnóstico de transtorno mental e o tratamento medicamentoso foram coletados a partir da consulta ao prontuário do usuário. Para avaliar o conhecimento dos participantes sobre o tratamento medicamentoso, as respostas fornecidas por eles quanto ao nome, a dose e a frequência de administração dos medicamentos foram contrastadas com a prescrição médica consultada em prontuário.

Considerando cada variável do tratamento medicamentoso (nome, dose e frequência), os participantes foram classificados com "nível de conhecimento suficiente” quando obtiveram aproveitamento total das respostas; ou, "nível de conhecimento insuficiente" quando não souberam responder uma ou mais perguntas.

Os dados coletados foram codificados e alimentados em um banco de dados do Microsoft Excel ${ }^{\circledR}$ e submetidos à dupla checagem de codificação e tabulação das questões. Os dados foram analisados com o auxílio do programa computacional Statistical Package for the Social Sciences (SPSS) 20ํㅡㄹ versão.

Os dados foram submetidos a análise descritiva e inferencial. $\mathrm{Na}$ comparação entre as variáveis do nível de conhecimento e a idade foi utilizado o Teste T de Student. Para a associação entre o nível de conhecimento e as variáveis qualitativas foi utilizado o teste Qui-quadrado ou o teste exato de Fisher, considerando valores de $\mathrm{p}<0,05$ significantes. Os resultados das variáveis 
quantitativas foram descritos por médias, desvios padrões, medianas, valores mínimos e valores máximos. Enquanto as variáveis qualitativas foram descritas por frequências e percentuais.

Este estudo obteve parecer positivo na data de 18 de setembro de 2013 pelo Comitê de Ética em Pesquisa da Universidade Federal do Paraná sob o protocolo no 406.158, CAAE: 20816713.9.0000.0102. Os preceitos éticos foram salvaguardados em consonância com a Resolução n 466, de 12 de dezembro de 2012 do Conselho Nacional de Saúde. Ainda, a participação da pesquisa foi consentida mediante a assinatura do Termo de Consentimento Livre e Esclarecido, sendo garantidos todos os aspectos éticos de acordo com a resolução 466/12 do Conselho Nacional de Saúde.

\section{Resultados}

Quanto às características sociodemográficas dos participantes: o sexo feminino teve prevalência de $63 \%$ ( $\mathrm{n}=189$ ); a idade média foi de 42,2 anos com desvio padrão de \pm 11 anos; também houve prevalência de solteiros em $44,7 \%$ ( $n=134)$; desempregados em $38,3 \%$ ( $n=115)$; com ensino médio completo em $38,7 \%(n=116)$, sequenciado por aqueles com ensino fundamental incompleto em $32,7 \%(\mathrm{n}=98)$.

Nas características clínicas predominou pessoas com diagnóstico de Transtorno Afetivo Bipolar em 33,5\% (n=123), Esquizofrenia em 19,1\% (n=70) e Depressão em 18,5\% (n=68). Quanto as classes medicamentosas prescritas de saúde mental, dentre os 777 medicamentos prescritos, houve o predomínio de antipsicóticos em 32,3\% (n=251), sequenciados de estabilizadores de humor em $22,8 \%(\mathrm{n}=177)$, de ansiolíticos e indutores do sono em $21,9 \%(\mathrm{n}=170)$ e antidepressivos em $20,4 \%$ ( $n=158)$. Destaca-se que 61\% ( $n=183)$ possuía alguma comorbidade clínica, destes $83,1 \%(n=152)$ referiram utilizar medicamentos clínicos.

Ao comparar as informações fornecidas pelos participantes sobre o seu tratamento medicamentoso com as informações sobre a prescrição de medicamentos presentes em 
prontuário, a maioria mostrou conhecimento insuficiente sobre o nome dos medicamentos, $62,3 \%$ $(\mathrm{n}=187)$ dos participantes referiu não saber informar o nome dos medicamentos. Os participantes também apresentaram conhecimento insuficiente quanto a dose dos medicamentos em 91,9\% ( $n=274)$ e a frequência de administração em $82,6 \%(n=247)$, conforme a Tabela 1.

Tabela 1- Caracterização do conhecimento das pessoas com transtorno mental sobre o nome do fármaco, a dose e a frequência de administração. Curitiba, Paraná. Brasil, 2019.

\begin{tabular}{llllllll} 
& & \multicolumn{2}{c}{ Feminino } & Masculino & \multicolumn{2}{c}{ Total } \\
\cline { 2 - 7 } Variável & $\mathrm{n}$ & $\%$ & $\mathrm{n}$ & $\%$ & $\mathrm{n}$ & $\%$ \\
\hline
\end{tabular}

Conhecimento sobre o(s) nome $(s)$ do(s) medicamentos

\begin{tabular}{|c|c|c|c|c|c|c|}
\hline Conhecimento insuficiente & 116 & 61,4 & 71 & 64 & 187 & 62,3 \\
\hline Conhecimento suficiente & 73 & 38,6 & 40 & 36 & 113 & 37,7 \\
\hline Total & 189 & 100 & 111 & 100 & 300 & 100 \\
\hline \multicolumn{7}{|c|}{ Conhecimento sobre a(s) dose $(s)$ dos medicamentos } \\
\hline Conhecimento insuficiente & 170 & 90,9 & 104 & 93,7 & 274 & 91.9 \\
\hline Conhece suficiente & 17 & 9,1 & 7 & 6,3 & 24 & 8,1 \\
\hline Total $^{*}$ & 187 & 100 & 111 & 100 & 298 & 100 \\
\hline \multicolumn{7}{|c|}{ Conhecimento sobre a frequência de administração do(s) medicamentos } \\
\hline Conhecimento insuficiente & 151 & 80,3 & 96 & 86,5 & 247 & 82,6 \\
\hline Conhecimento suficiente & 37 & 19,7 & 15 & 13,5 & 52 & 17,4 \\
\hline Total $^{*}$ & 188 & 100 & 111 & 100 & 299 & 100 \\
\hline
\end{tabular}

*Houve perdas por ausência de informações no prontuário para realizar a comparação com as informações fornecidas pelos participantes.

Ao comparar o conhecimento dos participantes quanto ao nome dos medicamentos com as variáveis sociodemográficas e clínicas, a análise estatística mostrou que o conhecimento insuficiente sobre o nome dos medicamentos foi mais provável de ocorrer entre usuários que utilizam medicamentos para outros problemas de saúde (p: 0,031), entre aqueles que não souberam informar o seu diagnóstico de transtorno mental (p:0,049), que possuem diagnóstico de depressão (p:0,016) e que apresentaram maior média de idade (p: 0,012). 
9 | Alcantara CB, Ferreira ACZ, Capistrano FC, Kaled K, Vale CCF, Maftum MA

Tabela 2 - Distribuição de variáveis sociodemográficas e clínicas das pessoas com transtornos mentais associadas ao conhecimento do(s) nome(s) do(s) medicamento(s). Curitiba, Paraná. Brasil, 2019.

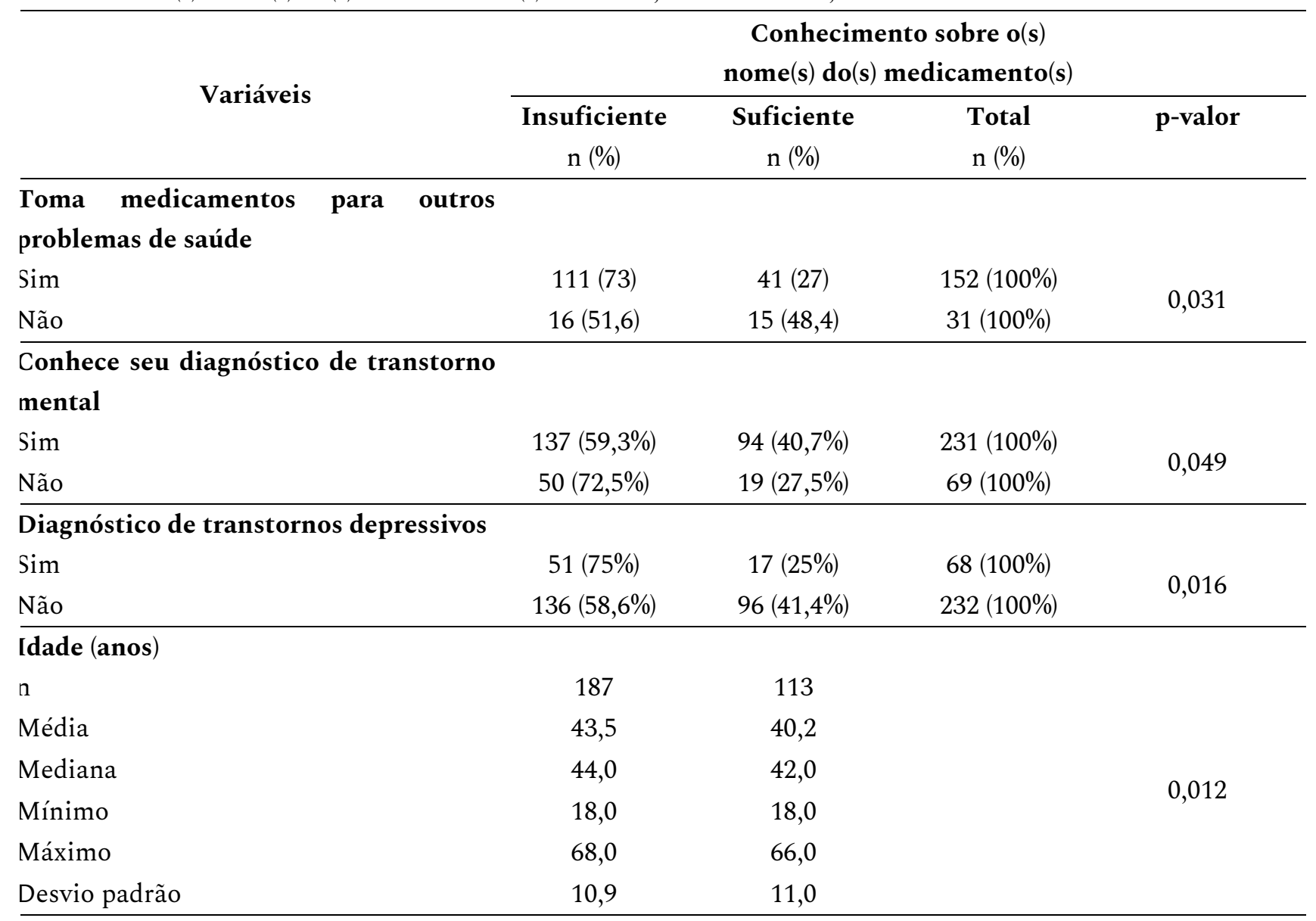

Em consonância com a Tabela 3, foi possível identificar, a partir de associação significativa, que a maioria dos participantes que apresentava diagnóstico de esquizofrenia (p: 0,042) e que realizava sozinho a autoadministração dos medicamentos (p: 0,017) apresentava conhecimento insuficiente sobre a dose dos medicamentos. Enfatiza-se que pessoas mais velhas, expressas por maior média de idade, foram mais propensas a apresentar conhecimento insuficiente (p: 0,004). 
Tabela 3 - Distribuição de variáveis sociodemográficas e clínicas das pessoas com transtornos mentais associadas ao conhecimento da(s) dose(s) do(s) medicamento(s). Curitiba, Paraná. Brasil, 2019.

\begin{tabular}{|c|c|c|c|c|}
\hline \multirow{2}{*}{ Variáveis } & \multicolumn{4}{|c|}{ Conhecimento sobre a $(s)$ dose $(s)$ do $(s)$ medicamento(s) } \\
\hline & $\begin{array}{c}\text { Insuficiente } \\
\mathrm{n}(\%)\end{array}$ & $\begin{array}{c}\text { Suficiente } \\
\mathrm{n}(\%)\end{array}$ & $\begin{array}{l}\text { Total } \\
\mathrm{n}(\%)\end{array}$ & p-valor \\
\hline \multicolumn{5}{|c|}{ Diagnóstico de Esquizofrenia } \\
\hline $\operatorname{sim}$ & $60(85,7 \%)$ & $10(14,3 \%)$ & $70(100 \%)$ & \multirow{2}{*}{0,042} \\
\hline Não & $214(93,9 \%)$ & $14(6,1 \%)$ & $228(100 \%)$ & \\
\hline \multirow{2}{*}{\multicolumn{5}{|c|}{$\begin{array}{l}\text { Realiza sozinho(a) a administração dos } \\
\text { medicamentos }\end{array}$}} \\
\hline & & & & \\
\hline $\operatorname{Sim}$ & $144(88,3 \%)$ & $19(11,7 \%)$ & $163(100 \%)$ & \multirow{2}{*}{0,017} \\
\hline Não & $130(96,3 \%)$ & $5(3,7 \%)$ & $135(100 \%)$ & \\
\hline \multicolumn{5}{|l|}{ Idade (anos) } \\
\hline $\mathrm{n}$ & 274 & 24 & & \multirow{6}{*}{0,004} \\
\hline Média & 42,8 & 36,0 & & \\
\hline Mediana & 44,0 & 35,5 & & \\
\hline Mínimo & 18,0 & 19,0 & & \\
\hline Máximo & 68,0 & 57,0 & & \\
\hline Desvio padrão & 10,9 & 10,9 & & \\
\hline
\end{tabular}

Conforme a Tabela 4, a maioria dos participantes que relatou consumir bebidas alcoólicas, mesmo que esporadicamente, apresentaram significativamente conhecimento insuficiente sobre a frequência de administração de medicamentos (p: 0,024).

Ressalta-se que $60 \%(\mathrm{n}=180)$ dos participantes mencionaram receber algum tipo de informação sobre o tratamento medicamentoso e, destes, 95\% $(n=171)$ receberam estas informações pela equipe médica, 45,5\% ( $\mathrm{n}=73)$ pela equipe de Enfermagem, 32,8\% $(\mathrm{n}=59)$ pelos psicólogos e 15\% $(\mathrm{n}=27)$ pelos assistentes sociais. Os participantes poderiam referir ter recebido orientações sobre o tratamento medicamentoso por mais de um profissional.

Considerando essas variáveis, houve uma associação significativa entre conhecimento sobre a frequência de administração, com maior prevalência de conhecimento insuficiente entre aqueles que receberam informações sobre o tratamento pela equipe de enfermagem (p: 0,011) e pelos psicólogos (p: 0,023). 
11 | Alcantara CB, Ferreira ACZ, Capistrano FC, Kaled K, Vale CCF, Maftum MA

Tabela 4 - Distribuição de variáveis sociodemográficas e clínicas das pessoas com transtornos mentais associadas ao conhecimento da(s) frequência(s) de administração do(s) medicamento(s). Curitiba, Paraná. Brasil, 2019.

\begin{tabular}{|c|c|c|c|c|}
\hline \multirow{3}{*}{ Variáveis } & \multicolumn{4}{|c|}{ Conhecimento sobre a frequência de administração do(s) } \\
\hline & \multicolumn{4}{|c|}{ medicamento(s) } \\
\hline & $\begin{array}{c}\text { Insuficiente } \\
\mathrm{n}(\%)\end{array}$ & $\begin{array}{c}\text { Suficiente } \\
\mathrm{n}(\%)\end{array}$ & $\begin{array}{l}\text { Total } \\
\text { n }(\%)\end{array}$ & p-valor \\
\hline \multicolumn{5}{|l|}{ Consumo de bebida alcoólica } \\
\hline Sim & $31(96,9 \%)$ & $1(3,1 \%)$ & $32(100 \%)$ & \multirow{2}{*}{0,024} \\
\hline Não & $216(80,9 \%)$ & $51(19,1 \%)$ & $267(100 \%)$ & \\
\hline \multirow{2}{*}{\multicolumn{5}{|c|}{$\begin{array}{l}\text { Informações sobre a terapêutica } \\
\text { medicamentosa recebidas pela Equipe } \\
\text { de Enfermagem }\end{array}$}} \\
\hline & & & & \\
\hline $\operatorname{Sim}$ & $66(90,4 \%)$ & $7(9,6 \%)$ & $73(100 \%)$ & \multirow{2}{*}{0,011} \\
\hline Não & $80(75,5 \%)$ & $26(24,5 \%)$ & $106(100 \%)$ & \\
\hline \multicolumn{5}{|l|}{ Informações $\quad$ sobre $\quad$ a terapêutica } \\
\hline medicamentosa recebidas pelos & & & & \\
\hline \multicolumn{5}{|l|}{ Psicólogos } \\
\hline $\operatorname{sim}$ & $54(91,5 \%)$ & $5(8,5 \%)$ & $59(100 \%)$ & \multirow{2}{*}{0,023} \\
\hline Não & $92(76,7 \%)$ & $28(23,3 \%)$ & $120(100 \%)$ & \\
\hline
\end{tabular}

\section{Discussão}

Neste estudo, a maioria das pessoas com transtornos mentais em tratamento em CAPS desconhece o nome, a dose e a frequência de administração dos medicamentos prescritos. Diferentemente, pesquisa desenvolvida com pessoas diagnosticadas com esquizofrenia em tratamento em um Núcleo de Saúde Mental de São Paulo, mostrou que 76,2\% dos participantes sabiam informar o nome dos medicamentos, enquanto $28,8 \%$ e $57,1 \%$ dos usuários desconheciam a dose e a frequência de administração, respectivamente. ${ }^{4}$

O pouco conhecimento de pessoas com transtornos mentais sobre o tratamento medicamentoso pode comprometer a autoadministração e representa um risco a segurança do usuário, pois o mesmo pode fazer o uso de doses maiores ou menores que aquelas prescritas, elevando riscos de intoxicação, acentuação dos efeitos colaterais e menor tolerabilidade. Deste modo, o uso 
incorreto de medicamentos para o tratamento em saúde mental pode comprometer significativamente a eficácia do tratamento, resultando em pior prognóstico e maior risco de (re)internações..$^{7,9,16}$

A literatura evidencia que a pessoa com transtorno mental pode apresentar limitações cognitivas decorrentes da manifestação do transtorno, afetando diretamente a memória, a atenção e a compreensão. Essas limitações podem comprometer o uso correto dos medicamentos e são agravadas diante de esquemas terapêuticos complexos. ${ }^{4,8}$

Além disto, este estudo evidenciou que as pessoas que utilizam medicamentos para outros problemas de saúde são mais propensas a apresentar conhecimento insuficiente sobre os nomes dos medicamentos para o transtorno mental. Acredita-se que a utilização de múltiplos fármacos pode levar a interações medicamentosas e a maior exposição a efeitos adversos e colaterais, além de tornar o tratamento mais complexo, exigindo do usuário maior atenção, memória e organização quanto a doses e horários de administração. ${ }^{17}$

A administração de múltiplos medicamentos quando associada à precária orientação e informação sobre o tratamento pode representar um desafio ao usuário, refletindo um baixo nível de conhecimento e, assim comprometer a adesão ao tratamento. ${ }^{7,16-17}$ Assim, a literatura evidencia que a simplificação dos esquemas terapêuticos favorece a utilização correta dos medicamentos e a melhora do quadro de saúde. ${ }^{18}$

O conhecimento insuficiente sobre o nome dos medicamentos foi associado ao desconhecimento do participante sobre o seu diagnóstico de transtorno mental. Semelhança a estes dados um estudo com pessoas com transtornos ansiosos em tratamento ambulatorial no interior de São Paulo evidenciou que o déficit de conhecimento sobre o transtorno acometido e o tratamento medicamentoso frequentemente dificultam a adesão ao tratamento psicossocial. Compreender o transtorno, a condição da saúde e o tratamento é considerado uma premissa para que o usuário 
internalize a necessidade da terapêutica medicamentosa e tenha motivação para utilizar corretamente os medicamentos. ${ }^{19}$

O usuário que desconhece o seu diagnóstico de transtorno mental e o tratamento proposto pode, consequentemente, não aderir ao tratamento por apresentar dúvidas frequentes quanto à necessidade e a efetividade do tratamento medicamentoso. ${ }^{4,8}$ Nesta pesquisa, o conhecimento insuficiente sobre o nome do medicamento apresentou associação com o diagnóstico de transtornos depressivos. Destaca-se que os sintomas depressivos expressos pela falta de motivação, letargia, déficit de concentração e dificuldade em tomadas de decisão, frequentemente, comprometem o desenvolvimento de atividades diárias, inclusive a terapêutica medicamentosa. ${ }^{20}$

Nesta perspectiva, uma pesquisa desenvolvida em um serviço de saúde mental ambulatorial, com 27 pessoas com transtornos depressivos, também evidenciou um baixo nível conhecimento sobre a maior parte do esquema terapêutico entre os participantes, o qual foi atribuído, por eles, à falta de atividades educativas em saúde pela equipe de saúde, a dificuldade de compreensão das informações recebidas pelos profissionais do serviço e a episódios de esquecimento. ${ }^{20}$

A maior média de idade se associou ao desconhecimento do nome e dose dos psicofármacos. Ressalta-se que o funcionamento cognitivo se degenera com a idade e, assim sendo a literatura evidencia que, frequentemente, usuários com idade mais avançada apresentam menores índices de conhecimento sobre sua prescrição medicamentosa. ${ }^{21-22}$ Nessa perspectiva, um estudo desenvolvido em farmácias comunitárias na Holanda, com 418 pessoas submetidas a algum tratamento medicamentoso, desvelou que pessoas com idade mais avançada são mais propensas a apresentar índices mais baixos de conhecimento sobre tal terapêutica. ${ }^{23}$

Ressalta-se que o conhecimento insuficiente sobre a dose dos medicamentos prescritos mostrou associação com o diagnóstico de esquizofrenia, resultados que vão ao encontro com o estudo realizado com 21 pessoas com esquizofrenia em tratamento em um serviço de saúde mental do 
Estado de São Paulo, que mostrou a prevalência de baixo conhecimento sobre a dose dos medicamentos prescritos pelos participantes em que $57,1 \%$ desconheciam a dosagem. ${ }^{4}$

A maioria dos participantes que verbalizou realizar a autoadministração dos medicamentos apresentou conhecimento insuficiente sobre a dose dos medicamentos. Contudo, a literatura evidencia que o conhecimento sobre o nome, a dose e a frequência dos medicamentos é a condição essencial para realizar o uso seguro de medicamentos para quem não possui a medicação supervisionada. ${ }^{9}$

As dificuldades das pessoas com transtorno mental em realizar a autoadministração dos psicofármacos intensificam o comportamento de não adesão ao tratamento medicamentoso de modo não intencional, favorecendo o descumprimento do uso regular, principalmente, quando há complexidade do esquema terapêutico e inexistência de supervisão de terceiros. ${ }^{9}$

Este estudo mostra que pessoas que referiram fazer o consumo de bebidas alcoólicas, ainda que de forma esporádica, apresentaram baixo conhecimento sobre a frequência de administração dos medicamentos. A literatura evidencia que o consumo de drogas lícitas e ilícitas favorece perdas episódicas de memória e déficits cognitivos quando estão sob efeito dessas substâncias, principalmente, ao considerar que possuem ação prioritária no sistema nervoso central. ${ }^{21}$

Estudo realizado com pessoas com transtornos mentais em CAPS de um município de Curitiba mostra que o uso de psicofármacos concomitante a bebidas alcoólicas é uma prática frequente nessa população, mesmo, conscientes dos riscos das interações entre as substâncias. Acredita-se que a partir dessa interação as pessoas buscam momentos de fuga de realidade, de intenso prazer, descontração e tranquilidade. ${ }^{8}$

Este estudo também mostra que os usuários que foram orientados sobre o tratamento medicamentoso pela equipe de enfermagem e por psicólogos apresentaram conhecimento insuficiente acerca da frequência de administração dos medicamentos. De forma semelhante, estudo desenvolvido com pessoas com transtorno mental em São Paulo evidenciou, a partir da 
percepção dos participantes, que quando o usuário recebia informações sobre o uso de medicamentos pela equipe multiprofissional, apresentavam dificuldade em compreendê-las ou em memorizar as informações recebidas. Deste modo, destacam a importância de maior qualificação e reavaliação das atividades educativas. ${ }^{20}$

Deste modo, a literatura evidencia a importância de a equipe de saúde realizar atividades terapêuticas voltadas para a educação em saúde e que promovam o protagonismo do usuário no seu tratamento, principalmente no que se refere ao tratamento medicamentoso. Ademais, destaca-se a importância de um acompanhamento contínuo do usuário com o objetivo de avaliar se as informações foram adequadamente compreendidas e, consequentemente, minimizar os riscos envolvidos e promover o uso seguro dos medicamentos. ${ }^{7,9,16}$

\section{Conclusão}

Este estudo identificou déficit de conhecimento sobre a prescrição de medicamentos, condição que pode resultar no uso irregular dos psicofármacos, comprometendo os resultados do tratamento e a segurança do usuário. Ademais, mostrou que o conhecimento insuficiente sobre o nome dos medicamentos estava associado ao uso de fármacos para condições clínicas, desconhecimento do diagnóstico de transtorno mental, transtornos depressivos e maior média de idade. A insuficiência de conhecimento sobre a dose se relacionou ao diagnóstico de esquizofrenia e autoadministração dos medicamentos, enquanto o conhecimento insuficiente sobre a frequência de administração se associou a receber orientações sobre o uso de psicofármacos pela equipe de enfermagem e por psicólogos.

Estes achados mostram que diversos fatores intrapessoais e interpessoais podem estar relacionados ao nível de conhecimento e representar obstáculos à construção do conhecimento sobre o tratamento medicamentoso. Deste modo, evidencia-se a necessidade da equipe de saúde contemplar em seu processo de trabalho a educação em saúde à pessoa com transtornos mentais 
acerca do uso de psicofármacos e, principalmente, a necessidade de se ater ao modo como as orientações são realizadas buscando adaptá-las às necessidades, nível educacional e funcionamento cognitivo da pessoa com transtorno mental e seus familiares.

Evidencia-se a necessidade de pesquisas, com maiores níveis de evidência, que explorem o conhecimento das pessoas com transtornos mentais sobre o tratamento medicamentoso, assim como os fatores que interferem neste processo e, que contemplem estratégias para intervir sobre as dificuldades de aprendizagem e memorização, objetivando a promoção do conhecimento, do uso seguro de fármacos e da adesão ao tratamento.

Ressalta-se que este estudo apresenta limitações em decorrência do delineamento transversal que não permite identificar a temporalidade das associações entre o conhecimento do tratamento medicamentoso e os fatores sociodemográficos, clínicos e terapêuticos. Este estudo limita os achados por abordar o uso de medicamentos de forma generalizada, sem estratificar as classes medicamentosas ou os diferentes transtornos mentais, assim como por não utilizar instrumentos validados para avaliar o conhecimento.

\section{Referências}

1. Organização Mundial da Saúde (OMS). Investing in mental health: evidence for action. Genebra: Organização Mundial da Saúde; 2013.

2. Ministério da Saúde (BR). Diretrizes Assistenciais em Saúde Mental Suplementar. Brasília (DF): Ministério da Saúde; 2008.

3. American Psychiatric Association (EUA). Manual diagnóstico e estatístico de transtornos mentais: DSM- 5. 5a ed. Porto Alegre (RS): Artmed; 2014.

4. Miasso AI, Miamoto CS, Mercedes BPC, Vedana KGG. Adesão, conhecimento e dificuldades relacionados ao tratamento farmacológico entre pessoas com esquizofrenia. Rev Eletrônica Enferm [Internet]. 2015 [acesso em 2019 abr 19];17(2):186-95. Disponível em: https://revistas.ufg.br/fen/article/view/27636 doi: https://doi.org/10.5216/ree.v17i2.27636 
5. Hiany N, Vieira MA, Gusmão ROM, Barbosa SFA. Perfil epidemiológico dos transtornos mentais na população adulta do Brasil: uma revisão integrativa. Rev Enferm Atual In Derme [Internet]. 2018 [acesso em 2019 abr 02];2018(86). Disponível em: http://revistaenfermagematual.com/arquivos/ED_86_REVISTA_24/42.pdf

6. Pinho LG, Pereira A, Chaves C. Adaptação portuguesa da escala de qualidade de vida para pessoas com esquizofrenia. Rev Iberoam Diagn Eval Psicol [Internet]. 2018 [acesso em 2019 abr 19];1(46):189-99. Disponível em: https://www.researchgate.net/publication/322320084 doi: 10.21865/RIDEP46.1.14

7. Alcântara CB, Capistrano FC, Czarnobay J, Ferreira ACZ, Brusamarello T, Maftum MA. Drug therapy for people with mental disorders in the view of nursing professionals. Esc Anna Nery Rev Enferm [Internet]. 2018 [acesso em 2019 abr 18];22(2):e20170294. Disponível em: http://www.scielo.br/scielo.php?script=sci_arttext\&pid=S1414-81452018000200201\&lng=en\&tlng=en doi: 10.1590/2177-9465-ean-2017-0294

8. Ferreira ACZ, Borba LO, Capistrano FC, Czarnobay J, Maftum MA. Fatores que interferem na adesão ao tratamento de dependência química: percepção de profissionais de saúde. REME Rev Min Enferm [Internet]. 2015 [acesso em 2019 abr 18];19(2):150-6. Disponível em: http://reme.org.br/artigo/detalhes/1012 doi: $10.5935 / 1415-2762.20150032$

9. Sousa LPC, Vedana KGG, Miasso AI. Adesão ao tratamento medicamentoso por pessoas com transtorno de ansiedade. Cogitare Enferm [Internet]. 2016 [acesso em 2019 abr 18];21(1):01-11. Disponível em: https://revistas.ufpr.br/cogitare/article/view/43510 doi: 10.5380/ce.v21i1.43510

10. Carvalho TR, Okuno MFP, Campanharo CRV, Lopes MCBT, Batista REA. Conhecimento dos pacientes sobre a prescrição medicamentosa no serviço de emergência. Rev Bras Enferm [Internet]. 2018 [acesso em 2019 abr 18];71(2):351-7. Disponível em: http://www.scielo.br/scielo.php?script=sci_arttext\&pid=S003471672018000200329\&lng=en\&nrm=iso\&tlng=pt doi: 10.1590/0034-7167-2017-0002

11. Fröhlic SE, Pizzol TSD, Mengue SS. Instrumento para avaliação do nível de conhecimento da prescrição na atenção primária. Rev Saúde Pública [Internet]. 2010 [acesso em 2019 abr 19];44(6):1046-54. Disponível em: http://www.scielo.br/scielo.php?script=sci_arttext\&pid=\$0034-89102010000600009 doi: 10.1590/S0034-89102010000600009

12. Melchiors AC, Correr CJ, Fernández-Llimos F. Tradução e validação para o português do Medication Regimen Complexity Index. Arq Bras Cardiol [Internet]. 2007 [acesso em 2019 abr 19];89(4):210-8. Disponível em: http://www.scielo.br/scielo.php?script=sci_arttext\&pid=S0066782X2007001600001\&lng=pt\&nrm=iso\&tlng=pt doi: 10.1590/S0066-782X2007001600001

13. Czarnobay J, Ferreira ACZ, Capistrano FC, Borba LO, Kalinke LP, Maftum MA. Determinantes intra e interpessoais percebidos pela família como causa da recaída do dependente químico. REME Rev Min 
Enferm [Internet]. 2015 [acesso em 2019 abr 18];19(2):93-9. Disponível em: http://www.reme.org.br/artigo/detalhes/1008 doi: 10.5935/1415-2762.20150028

14. Organização Pan-Americana da Saúde (OPAS-BR), Organização Mundial da Saúde (OMS). Uso racional de medicamentos: fundamentação em condutas terapêuticas e nos macroprocessos da assistência farmacêutica. Brasília (DF): Organização Pan-Americana da Saúde; 2015. Disponível em: https://www.paho.org/bra/index.php?option=com_docman\&view=document\&layout=default\&alias=1546administracao-nao-basta-usar-e-preciso-conhecer-a-maneira-correta-6\&category_slug=serie-uso-racionalmedicamentos-284\&format=html\&Itemid=965

15. Gazzinelli MF, Souza V, Fonseca RMGS, Fernandes MM, Carneiro ACLL, Godinh LK. Práticas educativas grupais na atenção básica: padrões de interação entre profissionais, usuários e conhecimento. Rev Esc Enferm USP [Internet]. 2015 [acesso em 2019 abr 18];49(2):284-91. Disponível em: http://www.scielo.br/scielo.php?pid=S0080-62342015000200284\&script=sci_abstract\&tlng=pt doi: 10.1590/S0080-623420150000200014

16. Gonçalves LLM, Campos RTO. Narrativas de usuários de saúde mental em uma experiência de gestão autônoma de medicação. Cad Saúde Pública [Internet]. 2017 [acesso em 2019 abr 19];33(11):e00166216. Disponível em: http://www.scielo.br/scielo.php?script=sci_arttext\&pid=S0102311X2017001105002\&lng=pt\&tlng=pt doi: 10.1590/0102-311x00166216

17. Ferreira ACZ, Brusamarello T, Capistrano FC, Marin MJS, Maftum MA. A vivência do portador de transtorno mental no uso de psicofármacos na perspectiva do pensamento complexo. Texto \& Contexto Enferm [Internet]. 2017 [acesso em 2019 abr 19];26(3):e1000016. Disponível em: http://www.scielo.br/scielo.php?script=sci_arttext\&pid=S0104-07072017000300306\&lng=pt\&tlng=pt doi: 10.1590/0104-07072017001000016

18. Sá BTP, Martins MR, Boger B, Dias FA, Rattmann YD. Estimativa de adesão ao medicamento antirretroviral conformulado 3 em 1. ABCS Health Sci [Internet]. 2018 [acesso em 2019 dez 11];43(3):136-40. Disponível em: https://portalnepas.org.br/abcshs/article/view/1002/816 doi: 10.7322/abcshs.v43i3.1002

19. Cruz LP, Vedana KGG, Mercedes BPC, Miasso AI. Dificuldades relacionadas à terapêutica medicamentosa no transtorno de ansiedade. Rev Eletrônica Enferm [Internet]. 2016 [acesso em 2019 abr 19]; 18:e1155. Disponível em: https://revistas.ufg.br/fen/article/view/32741 doi: 10.5216/ree.v18.32741

20. Ibanez G, Mercedes BPC, Vedana KGG, Miasso AI. Adesão e dificuldades relacionadas ao tratamento medicamentoso em pacientes com depressão. Rev Bras Enferm [Internet]. 2014 [acesso em 2019 abr 19];67(4): 556-62. Disponível em: http://www.scielo.br/scielo.php?script=sci_arttext\&pid=S003471672014000400556 doi: 10.1590/0034-7167.2014670409

21. Marin MJS, Maftum MA, Lacerda MR. Idosos com transtornos mentais: vivenciando o uso de psicofármacos. Rev Bras Enferm [Internet]. 2018 [acesso em 2019 abr 19];71(2):888-96. Disponível em: 
19 | Alcantara CB, Ferreira ACZ, Capistrano FC, Kaled K, Vale CCF, Maftum MA

scielo.br/scielo.php?pid=S0034-71672018000800835\&script=sci_arttext\&tlng=pt doi: 10.1590/0034-71672016-0159

22. Silva ER, Ferreira ACZ, Borba LO, Kalinke LP, Nimtz MA, Maftum MA. Impacto das drogas na saúde física e mental de dependentes químicos. Ciênc Cuid Saúde [Internet]. 2016 [acesso em 2019 abr 19];15(1): 101-8. Disponível em: http://periodicos.uem.br/ojs/index.php/CiencCuidSaude/article/view/27137 doi: 10.4025/cienccuidsaude.v15i1.27137

23. Mertens BJ, Kwint HF, Van Marum RJ, Bouvy ML. Are multidose drug dispensing systems initiated for the appropriate patients? Eur J Clin Pharmacol [Internet]. 2018 [acesso em 2019 abr 19];74(9):1159-64. Disponível em: https://www.ncbi.nlm.nih.gov/pubmed/29770839 doi: 10.1007/s00228-018-2478-5

\section{Autor correspondente}

Camila Bonfim de Alcantara

E-mail: cbalcantara47@gmail.com

Endereço: Travessa Ema Moro, n.15 - Bairro Pedro Moro, São José dos Pinhais, Paraná, Brasil. CEP: 83005605

\section{Contribuições de Autoria}

1 - Camila Bonfim de Alcantara

Redação do manuscrito; Análise e interpretação dos dados.

2 - Aline Cristina Zerwes Ferreira

Concepção e planejamento do projeto de pesquisa; Coleta de dados; Análise e interpretação dos dados; revisão crítica da redação, dos resultados e da discussão dos dados.

3 - Fernanda Carolina Capistrano

Concepção e planejamento do projeto de pesquisa; Coleta de dados; revisão crítica da redação, dos resultados e da discussão dos dados.

4 - Manuela Kaled

Contribuições: revisão crítica da redação, dos resultados e da discussão dos dados.

5 - Caio Cézar da Fonseca Vale

Contribuições: revisão crítica da redação, dos resultados e da discussão dos dados.

6 - Mariluci Alves Maftum

Concepção e planejamento do projeto de pesquisa; Orientação e supervisão durante a coleta dos dados; Revisão crítica da redação, dos resultados e da discussão dos dados. 
Conhecimento da pessoa com transtornos mentais sobre o tratamento medicamentoso $\mid 20$

\section{Como citar este artigo}

Alcantara CB, Ferreira ACZ, Capistrano FC, Kaled K, Vale CCF, Maftum MA. Conhecimento da pessoa com transtornos mentais sobre o tratamento medicamentoso. Rev. Enferm. UFSM. 2020 [Acesso em: Anos Mês Dia]; vol.10 e24: 1-20. DOI:https://doi.org/10.5902/2179769238607 